\title{
Reaching with alien limbs: Visual exposure to prosthetic hands in a mirror biases proprioception without accompanying illusions of ownership
}

\author{
NICHOLAS P. HOLMES \\ University of Oxford, Oxford, England \\ HENDRIKUS J. SNIJDERS \\ University of Oxford, Oxford, England \\ and Utrecht University, Utrecht, The Netherlands \\ and \\ CHARLES SPENCE \\ University of Oxford, Oxford, England
}

\begin{abstract}
In five experiments, we investigated the effects of visual exposure to a real hand, a rubber hand, or a wooden block on reaching movements made with the unseen left hand behind a parasagittal mirror. Participants reached from one of four starting positions, corresponding to four levels of conflict between the proprioceptively and visually specified positions of the reaching hand. Reaching movements were affected most by exposure to the real hand, intermediately by the rubber hand, and least of all by the wooden block. When the posture and/or movement of the visible hand was incompatible with that of the reaching hand, the effect on reaching was reduced. A "rubber hand illusion" questionnaire revealed that illusions of ownership of the rubber hand were not strongly correlated with reaching performance. This research suggests that proprioception is recalibrated following visual exposure to prosthetic hands and that this recalibration is independent of the rubber hand illusion.
\end{abstract}

In order to reach toward a visible target, the brain needs to know the initial position of the reaching hand so that the correct movement direction and distance can be encoded (see, e.g., Rossetti, Desmurget, \& Prablanc, 1995). When reaching for objects in the dark, or when an arm is hidden from view, we must rely on proprioception to specify the initial position of the hand in relation to the target. If, instead of reaching "blind," we are provided with some visual information about the initial position of the hand, such as a brief glimpse of it or a visual marker signifying its position, proprioception and subsequent reaching movements may then be more accurate (see, e.g., Desmurget, Rossetti, Jordan, Meckler, \& Prablanc, 1997; Newport, Hindle, \& Jackson, 2001; Rossetti et al., 1995; Rossetti, Stelmach, Desmurget, Prablanc, \& Jeannerod, 1994; Wann \& Ibrahim, 1992; but see Bédard \& Proteau, 2001, and Sarlegna et al., 2004, for alternative views). Visual information for updating the felt position of the hands can also be provided by using a mirror aligned with the body midline,

N.P.H. was supported by a Wellcome Prize Studentship (065696/Z/01/ A) from the Wellcome Trust and a Science Research Fellowship from the Royal Commission for the Exhibition of 1851. Correspondence regarding this article should be addressed to N. P. Holmes, Espace et Action, INSERM Unité 534, 16 Avenue du Doyen Lepine, Bron, 69500 France (nicholas.p.holmes@wolfson.oxon.org). projecting the reflection of one hand into the apparent position of the other (Altschuler et al., 1999; Franz \& Packman, 2004; Holmes, Crozier, \& Spence, 2004; Holmes \& Spence, 2005; Ramachandran \& Rogers-Ramachandran, 1996; Ramachandran, Rogers-Ramachandran, \& Cobb, 1995; Ro, Wallace, Hagedorn, Farnè, \& Pienkos, 2004; Sathian, Greenspan, \& Wolf, 2000). When the two hands are aligned equidistantly from the mirror on either side, vision and proprioception provide complementary evidence concerning the position of the unseen hand. However, when the hands are placed at unequal distances from the mirror, the visually and proprioceptively specified hand positions are inconsistent with each other.

Mirror-induced conflicts between vision and proprioception have been shown to exert a bias on subsequent reaching movements made with the hand hidden behind the mirror (Holmes et al., 2004; Holmes \& Spence, 2005; see also Burnett, 1904; Nielsen, 1963; and Ro et al., 2004, for related effects of mirror-induced multisensory conflicts). Our previous work suggests that this reaching bias is caused, prior to the onset of the reaching movement itself, by a gradual process of recalibration of the proprioceptively specified position of the hand toward the visually specified position (Holmes \& Spence, 2005). This process seems to be incremental but quite rapid, since we found that reaching errors increased with increases in the duration of visual exposure to the incompatible hand posi- 
tion, from very little bias (in comparison with a no-mirror control condition) after $4 \mathrm{sec}$ of exposure to stronger and significant biases after 8 and $12 \mathrm{sec}$ of exposure (Holmes $\&$ Spence, 2005).

Although it is known that this reaching bias occurs when participants view their own, nonreaching hand reflected in a mirror, the critical sensory information sufficient to induce this bias is unknown. If the critical information is visual in nature, a visually presented object that looks similar to one's own hand might be sufficient to induce a spatial bias in reaching movements. If the critical information is postural or proprioceptive, however, reaching biases may only occur when a participant's two hands are positioned approximately symmetrically about the mirror (i.e., there may be some contribution of bilateral proprioceptive information to the apparently visual mirror illusion). Finally, if the critical information is indeed visual, one might predict that the more the visual input resembles the participant's real hand, the more reaching will be biased. Under this prediction, vision of a prosthetic hand, for example, will induce more reaching bias than will vision of a hand-sized block of wood. In order to investigate the sensory information required to induce such a bias in reaching movements, it may therefore be informative to expose participants to mirror reflections of their own nonreaching hand, an artificial rubber hand, or a block of wood of similar dimensions to their hands and to examine the effect of such exposure on subsequent reaching movements.

Stimuli such as prosthetic hands have been used frequently in recent years to investigate multisensory aspects of body representation and ownership (Armel \& Ramachandran, 2003; Austen, Soto-Faraco, Enns, \& Kingstone, 2004; Botvinick \& Cohen, 1998; Ehrsson, Spence, \& Passingham, 2004; Farnè, Pavani, Meneghello, \& Làdavas, 2000; Kennett, Taylor-Clarke, \& Haggard, 2001; Niebauer, Aselage, \& Schutte, 2002; Pavani, Spence, \& Driver, 2000; Peled, Pressman, Geva, \& Modai, 2003; Peled, Ritsner, Hirschmann, Geva, \& Modai, 2000; Rorden, Heutink, Greenfield, \& Robertson, 1999; Tsakiris \& Haggard, 2005; see also Tastevin, 1937). When artificial rubber hands are placed in a position that is compatible with a participant's current posture (i.e., in an anatomically plausible position with respect to the participant's own body), visual stimulation of the rubber hand coupled with tactile stimulation of the participant's own hand (which is hidden from view) induces, in many people, the illusory feeling that the visible rubber hand is the participant's own hand (Botvinick \& Cohen, 1998; Ehrsson et al., 2004). Such "rubber hand illusions" are eliminated or substantially reduced (in persistence or intensity) when the visual and tactile stimuli are applied asynchronously to the two hands, or when the rubber hand is placed in an anatomically implausible posture.

In the rubber hand illusion, participants are typically asked to rate the strength of the illusion using a visual scale to indicate the strength of their agreement with a variety of questionnaire statements. However, since such questionnaire ratings may be subject to experimental bi- ases or demand effects, experimenters have often also included a postillusion reaching and pointing response, in which participants point with one hand (the unexposed or nonstimulated hand) to the felt position of the other hand (the exposed hand). Typically, the strength or persistence of the illusion as determined from the questionnaire ratings is strongly correlated with errors in the postexposure pointing responses (Botvinick \& Cohen, 1998). These pointing errors have been taken to provide a measure of proprioceptive changes in the exposed arm and hand, and therefore a more objective measure of the rubber hand illusion itself than the subjective reports concerning the illusory experience.

Despite the use of postexposure pointing as an objective correlate of the rubber hand illusion, it has yet to be determined whether such shifts in the felt position of the exposed hand represent a cause, a consequence, or an epiphenomenal correlate of the rubber hand illusion itself. If recalibration of proprioception following exposure to a rubber hand either is a necessary prerequisite or is causally unrelated to the rubber hand illusion, then it ought to be possible to induce proprioceptive changes (and therefore to affect subsequent reaching movements) without inducing the illusion itself. Alternatively, if proprioceptive changes are a consequence of the rubber hand illusion, then any changes in proprioception following visual exposure to rubber hands should be strongly associated with the illusory experience of ownership of the rubber hand.

The aims of the experiments reported here were first to try to resolve the issue of the nature of the sensory information that is critical or sufficient to induce or enhance reaching biases in the mirror illusion, and second to determine whether viewing a rubber hand prior to executing a reaching movement can exert significant reaching biases in the absence of any deliberate attempt to induce (on the part of the experimenter) or to experience (on the part of the participant) a rubber hand illusion.

\section{EXPERIMENT 1}

In Experiment 1, we examined the influence of prior visual exposure to the reflection of a prosthetic rubber hand on the endpoints of subsequent reaching and pointing movements, as an index of the felt location of the hand immediately prior to the movement. We compared the effects of visual exposure to a real hand, a rubber hand, and a block of wood. If reaching movements are biased equally by prior visual exposure to any visually presented object in the approximate location of the participant's own reaching hand, there should be no difference between reaching movements under these three visual exposure conditions (the visual object hypothesis). By contrast, if prior exposure to very precise visual information or to near-bilateral symmetry of the participant's arms is required for the reaching bias to emerge, only the real-hand condition should induce strong reaching biases (the real-hand or bilateral proprioceptive hypothesis). Finally, if prior exposure to only approximate visual information concerning hand position is required, and if bilateral proprioceptive 
information is not critical, reaching in the rubber hand condition should be biased more than in the wooden block condition (the rubber hand hypothesis).

\section{Method}

Participants. Eighteen right-handed participants (19-32 years of age, 11 female), recruited by advertisement from the general population of students, staff, and visitors to Oxford University, took part in Experiment 1. The participants were either reimbursed $£ 5$ (UK sterling) for their time, or else participated for course credit. The experiment was approved by the local ethics committee, and the participants gave their informed consent prior to participation.

Materials. A rectangular mirror $(30 \times 45 \mathrm{~cm}$, see Figure 1$)$ was positioned vertically on a table $(62 \times 122 \mathrm{~cm})$ with the reflective surface facing to the right (all directions are given with respect to the participant, unless otherwise specified), to the immediate right of a platform $(45 \times 45 \mathrm{~cm}$, raised $20 \mathrm{~cm}$ off the table). Under the platform, $30 \mathrm{~cm}$ from the near edge of the table, four left hand position marks were drawn onto a sheet of paper: $7.5,12.5,17.5$, and $22.5 \mathrm{~cm}$ to the left of the mirror's reflective surface. A similar mark, representing the target position, was drawn $15 \mathrm{~cm}$ to the left of the mirror and $50 \mathrm{~cm}$ from the near edge of the table. Two further marks were positioned $15 \mathrm{~cm}$ to the right of the mirror: The first was $30 \mathrm{~cm}$ from the near edge of the table and served as the right hand position mark (and also marked the position for the index finger of the rubber hand and the corner of the wooden block in those conditions). The second was $50 \mathrm{~cm}$ from the near edge of the table and provided a virtual target position when viewed in the mirror. A 1-m square opaque black cloth was attached to the nearest side of the platform, covering the participant's left arm and shoulder. A small curved screen was constructed from a $21 \times 29 \mathrm{~cm}$ sheet of white paper and was positioned $22.5 \mathrm{~cm}$ to the right of the mirror at a slight angle away from it, approximately parallel to the participant's arm and the rubber hand or wooden block, and positioned about $30 \mathrm{~cm}$ from the mirror surface. When participants placed their right arm and hand behind this screen, their arm and hand were not visible in the mirror. The table was covered with white paper to provide a homogenous background surface.
Three exposure objects were used: the participant's real right hand; an artificial, realistic looking prosthetic right hand (approximately $16 \mathrm{~cm}$ from fingertips to wrist plus approximately $4 \mathrm{~cm}$ of forearm, $9 \mathrm{~cm}$ wide, $5 \mathrm{~cm}$ high); and a block of $\operatorname{wood}(7 \times 4.5 \times 20 \mathrm{~cm})$. The rubber hand had a smooth, Caucasian complexion similar to that of a female's hand and corresponded in size and shape approximately to the hand of a $155-\mathrm{cm}$-tall female. The skin characteristics of participants (i.e., color, hairiness, the presence of any spots or blemishes, etc.) were not controlled, measured, or selected for in any way, as has been typical in other studies using rubber hands (Botvinick \& Cohen, 1998; Ehrsson et al., 2004). The experimenter did, however, record whether each participant was broadly "white" or "nonwhite" in skin color in order to assess post hoc any between-participants effects of skin color. The experimenter used a stopwatch to time the visual exposure duration, and sheets of graph paper and four differently colored pens to mark the landing positions of reaching movements.

Design. The experiment followed a within-participants repeated measures design, with the independent variables left hand position $(7.5,12.5,17.5$, and $22.5 \mathrm{~cm})$ and visual exposure condition (real hand, rubber hand, and wooden block). The three experimental conditions were run in blocks of trials presented in a fully counterbalanced order across participants. In each block, the four left hand positions were presented five times each in a randomized order.

Procedure. The participants were asked to sit at the table and place their left arm behind the mirror. The cloth was draped over their left arm and shoulder to prevent direct vision of these body parts. The participants then placed their right arm behind the paper screen and looked into the mirror at the reflection of the target position. Before the experiment itself began, the participants were given practice at reaching to the target behind the mirror, attempting to place their left index finger on the target position. Verbal feedback (e.g., "too far left" or "too far right") was provided on the accuracy of these practice reaches, and the experimenter moved each participant's finger onto the target for additional feedback. As soon as the participants were able to reach consistently (i.e., for at least two successive trials) to within $2 \mathrm{~cm}$ of the target location, the main experimental session began (a circle $2 \mathrm{~cm}$ in diameter and centered

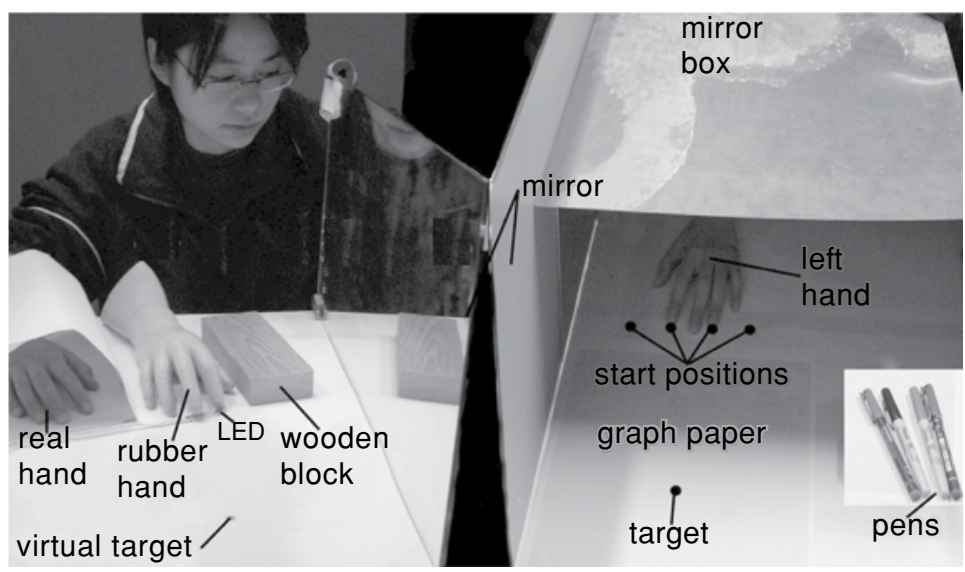

Figure 1. Experimental apparatus as seen from the experimenter's viewpoint. The participants sat with the right hand either behind the paper screen (as shown) or in the same position as the rubber hand. The participant's left hand was positioned inside the mirror box, shielded from view by the opaque cloth and mirror. The wooden block is shown next to the rubber hand for illustrative purposes only, and was moved into the position of the rubber hand during testing. The LED was used for Experiment 5 only, to serve as a fixation point and visual target. In Experiments 1-4, participants fixated the mirror reflection of the index finger of the rubber hand, their real hand, or the far left corner (from their perspective) of the wooden block. 
on the target was printed on the graph paper for assessing accuracy in the practice phase).

Each experimental block began with the experimenter placing the participant's left hand onto the target as an initial reminder of the (proprioceptive) target location. Next, the experimenter placed the exposure object onto the right hand position mark. In the realhand condition, this object was the index finger of the participant's right hand. In the rubber hand condition, the index finger of the rubber right hand was placed on the right hand mark, and the participant's right hand was placed behind the paper screen. The rubber hand was oriented with its palm facing down (i.e., matching the posture of both of the participant's hands). In the wooden block condition, the far left corner of the block (from the participant's point of view) was positioned on the right hand mark.

Each trial began with the experimenter moving the participant's left index finger (from the target on the first trial of each block, or from the previous reaching endpoint on all subsequent trials) to one of the left hand position marks, according to a predetermined pseudorandomized sequence. The participant was then instructed to fixate the reflection of the exposure object in the mirror, looking leftward toward the index finger of their own or the rubber hand, or toward the far left end of the wooden block (i.e., toward an apparent position $15 \mathrm{~cm}$ to the left of the mirror and $30 \mathrm{~cm}$ in front of the front edge of the table). This fixation position and posture were constant across visual exposure conditions and were maintained for $12 \mathrm{sec}$ while the experimenter monitored with a stopwatch to ensure the participant's visual fixation. The experimenter then gave the verbal instruction "Reach," at which point the participant made an eye movement to gaze at the (reflected) target location $20 \mathrm{~cm}$ to the front of the fixation position and reached with the left hand, making a single, smooth, uncorrected, rapid movement as accurately as possible toward the target position (i.e., as if seen "through" the mirror). The participants were instructed to gaze toward the virtual target location just prior to and during the reaching movement, to leave their index finger at the position where it first touched the table behind the mirror, and not to make any endpoint corrections after touching down on the table surface.

The experimenter marked the landing position of the participant's left index finger on the graph paper using a different-color pen for each starting position. Since we were interested only in errors made in the left-right $(x)$ direction, for the sake of clarity the experimenter occasionally offset some endpoint marks in the front-back $(y)$ direction in order to avoid superimposing endpoint marks on top of each other. The participant's index finger was then returned to the starting position for the next trial. No feedback on target pointing accuracy was provided before, during, or after the individual trials or for the experimental conditions as a whole. The participants were given a short break after each block of trials, during which time the experimenter changed the graph paper and visual exposure object in preparation for the next condition.

The layout of the left hand position marks, the right hand position mark, and the target location meant that the conflict induced between the visually and the proprioceptively specified left hand positions was, from right to left, $-7.5 \mathrm{~cm}$ (the $7.5-\mathrm{cm}$ left hand position mark), $-2.5 \mathrm{~cm}$ (the $12.5-\mathrm{cm}$ mark), $+2.5 \mathrm{~cm}$ (the $17.5-\mathrm{cm}$ mark), and $+7.5 \mathrm{~cm}$ (the $22.5-\mathrm{cm}$ mark), where positive values represent real (proprioceptive) left hand positions to the left of the apparent (visual) left hand position, and negative values are to the right of the apparent position. In all cases, the apparent position of the left hand suggested a straight-ahead reaching movement to meet the target, whereas all the actual left hand positions required a diagonal reach to either the right (the 7.5- and $12.5-\mathrm{cm}$ starting positions) or the left (the 17.5- and 22.5-cm starting positions). Visual biases of the reaching movements would therefore be evident when the reaching movements were too straight, thus falling short of the target position on the same side as the starting position.

It is important to note that the visual target location was always present and clearly visible throughout all blocks of trials, and the reaching task and hand positions were identical between visual exposure conditions. Furthermore, the participants were only instructed to make a quick reaching movement toward the visible target following the reach command, and at no point were they encouraged or directed by the experimenter to think about the felt location of their hand, or to try to remember the initial location of their hand at the start of the exposure period. The constant presence of the visual target ensured that the participants did not need to remember the proprioceptively specified location of the target, since the task required only a rapid uncorrected reaching and pointing movement from the current location of the hand at the end of the exposure period toward the visual target.

\section{Results}

Analysis. The endpoints of the reaching movements were recorded in one dimension only, the left-right or $x$ dimension perpendicular to the plane of the mirror. Reaches were measured on the graph paper to the nearest millimeter. Reaching errors to the left of the target were assigned a positive endpoint error, and those to the right were assigned negative values. The mean (constant error) and standard error (variable error) of the mean reaching error across participants were entered into a two-way repeated measures ANOVA with the within-participants variables left hand position and exposure condition. These data are displayed graphically in Figure 2, and the ANOVA statistics are displayed in Table 1. Separate analyses were performed with the additional variables block order, gender, and skin color to assess for practice and betweenparticipants effects, respectively. To provide an intuitive measure of the size of the visual bias effect, the slope of the best-fit linear regression equation that described the dependence of the endpoint errors on the starting position was calculated and expressed as a percentage (for example, a regression slope of .3 would correspond to a $30 \%$ reaching bias, or a $30 \%$ weighting of vision, and to $70 \%$ weighting of proprioception in producing the reaching movement). Unless stated otherwise, the data reported in all cases are the across-participants means \pm standard errors of the individual mean reaching errors.

Constant error. The upper panel of Figure 2 shows the mean and standard error across participants of the mean constant terminal reaching error, broken down by starting position and visual exposure condition. In general, reaching movements were biased by $1.6 \pm 0.2 \mathrm{~cm}$ toward the right side of the workspace - that is, toward the mirror. This general bias was similar between visual exposure conditions (real hand, $-1.6 \pm 0.3 \mathrm{~cm}$; rubber hand, $-1.6 \pm 0.4 \mathrm{~cm}$; wooden block, $-1.5 \pm 0.4 \mathrm{~cm}$ ) and is probably attributable to the asymmetrical posture that participants adopted in order to see their hand in the mirror: The participants' body midline was slightly to the right of the mirror, and unseen reaching movements often show general biases toward the midline (see, e.g., Ghilardi, Gordon, \& Ghez, 1995). In addition, reaching movements were in general too short, thus underestimating the lateral distance to the target. This is reflected in the significant main effect of left hand position and in the slope of the graphs across starting positions for each condition. Most importantly, however, the size of this endpoint bias (re- 


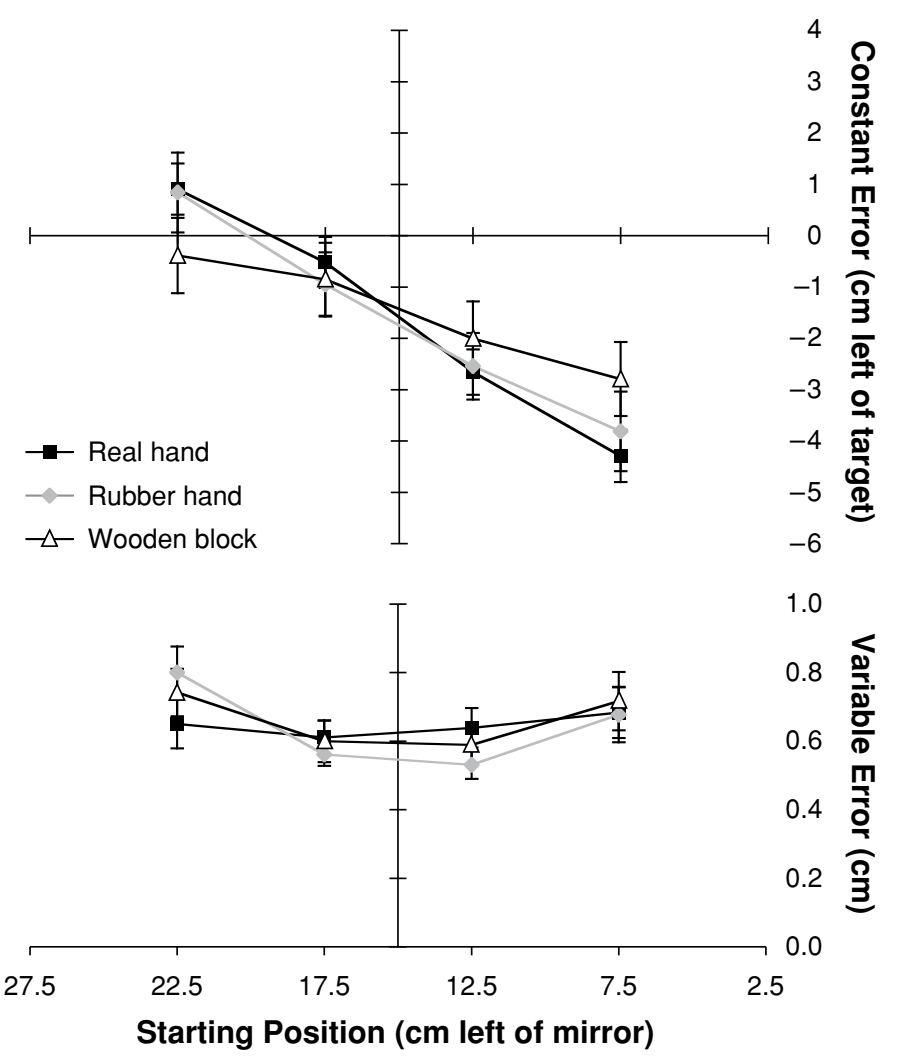

Figure 2. Mean ( $\pm S E$ ) endpoint errors for reaching movements in Experiment 1. Left hand starting positions are displayed from right to left, as if viewed from the participant's perspective. Top: Constant reaching error ( $M \pm S E$ of the individual mean reaching errors). Bottom: Variable reaching error $(M \pm S E$ of the standard error of the individual mean reaching errors).

flected in the gradient of each slope in Figure 2) depended significantly upon the visual exposure condition (i.e., a significant interaction occurred between left hand position and exposure condition). Reaching movements following visual exposure to a real hand were significantly more biased than those following exposure to the wooden block. In addition, exposure to the rubber hand also resulted in significantly stronger bias than did the wooden block condition. Separate ANOVAs comparing all pairings of the three conditions confirmed that there was no significant difference between reaching behavior in the real-hand versus the rubber hand condition [interaction of left hand position $\times$ exposure condition: $F(3,51)=$ $1.94, p=.14$ ], but reaching errors in both of these conditions differed significantly from the wooden block condition [real hand vs. wooden block, $F(3,51)=16.66, p<$ .001 ; rubber hand vs. wooden block, $F(3,51)=8.20, p<$ $.001]$. The reaching errors, expressed as a percentage of the lateral distance from the starting position to the target (derived from the linear regression slope relating the start positions with their respective mean endpoint errors across participants) were $35.5 \% \pm 2.3 \%$ in the real-hand condition, $31.1 \% \pm 3.4 \%$ in the rubber hand condition, and $16.7 \% \pm 3.0 \%$ in the wooden block condition.
Variable error. The variable error (the standard error of the mean reaching error per participant) data are displayed in the lower panel of Figure 2, and the ANOVA statistics are presented in Table 1. There were no significant effects of, or interactions involving, the visual exposure condition, since variability was approximately equal across the three conditions (real hand, $0.65 \pm 0.03 \mathrm{~cm}$; rubber hand, $0.64 \pm 0.03 \mathrm{~cm}$; wooden block, $0.66 \pm 0.03 \mathrm{~cm}$ ). Reaching endpoint variability was, however, significantly affected by the left hand position. Reaching movements from the two outermost starting positions were more variable than those from the two innermost starting positions (for $7.5 \mathrm{~cm}, 0.69 \pm 0.04 \mathrm{~cm}$; for $12.5 \mathrm{~cm}, 0.58 \pm 0.04 \mathrm{~cm}$; for $17.5 \mathrm{~cm}, 0.59 \pm 0.03 \mathrm{~cm}$; for $22.5 \mathrm{~cm}, 0.73 \pm 0.03 \mathrm{~cm}$ ).

\section{Discussion}

These results demonstrate that reaching movements were biased to a similar extent by $12 \mathrm{sec}$ of visual exposure to an artificial and to a real hand, although there was a trend toward a greater visual bias following exposure to the participant's real hand. Passive exposure to a wooden block, by contrast, had a smaller effect on subsequent reaching movements than did exposure to either the real hand or the rubber hand. These results favor the rubber 
Table 1

ANOVA Statistics for Experiments 1, 2, 4, and 5

\begin{tabular}{|c|c|c|c|c|c|c|c|c|c|c|}
\hline \multirow[b]{2}{*}{ Experiment } & \multirow[b]{2}{*}{ Measure } & \multicolumn{3}{|c|}{ Left Hand Position } & \multicolumn{3}{|c|}{ Exposure Condition } & \multicolumn{3}{|c|}{$\begin{array}{c}\text { Left Hand Position } \times \\
\text { Exposure Condition }\end{array}$} \\
\hline & & $d f$ & $F$ & $p$ & $d f$ & $F$ & $p$ & $d f$ & $F$ & $p$ \\
\hline \multirow[t]{2}{*}{1} & $\mathrm{CE}$ & 3,51 & 100.25 & $<.001$ & 2,34 & 0.03 & .98 & 6,102 & 9.21 & $<.001$ \\
\hline & VE & 3,51 & 6.13 & $<.001$ & 2,34 & 0.14 & .87 & 6,102 & 0.84 & .54 \\
\hline \multirow[t]{2}{*}{2} & $\mathrm{CE}$ & 3,60 & 71.70 & $<.001$ & 2,40 & 0.35 & .71 & 6,120 & 4.38 & $<.01$ \\
\hline & VE & 3,60 & 1.71 & .18 & 2,40 & 4.73 & $<.05$ & 6,120 & 1.49 & .19 \\
\hline \multirow[t]{2}{*}{4} & CE & 3,33 & 147.42 & $<.001$ & 2,22 & 0.46 & .64 & 6,66 & 7.38 & $<.001$ \\
\hline & VE & 3,33 & 2.34 & .09 & 2,22 & 0.03 & .97 & 6,66 & 0.33 & .92 \\
\hline \multirow[t]{6}{*}{5} & $\mathrm{CE}(x)$ & 3,33 & 24.70 & $<.001$ & 1,11 & 0.97 & .35 & 3,33 & 11.50 & $<.001$ \\
\hline & $\mathrm{VE}(x)$ & 3,33 & 7.77 & $<.001$ & 1,11 & 0.62 & .45 & 3,33 & 1.15 & .34 \\
\hline & $\mathrm{CE}(y)$ & 3,33 & 3.02 & $<.05$ & 1,11 & 0.81 & .39 & 3,33 & 1.79 & .17 \\
\hline & $\operatorname{VE}(y)$ & 3,33 & 0.39 & .76 & 1,11 & 0.07 & .79 & 3,33 & 0.52 & .67 \\
\hline & RT & 3,33 & 0.56 & .64 & 1,11 & $<0.01$ & .96 & 3,33 & 0.90 & .45 \\
\hline & Errors & 3,33 & 2.76 & .06 & 1,11 & 0.80 & .39 & 3,33 & 1.31 & .29 \\
\hline
\end{tabular}

Note-Statistics are presented for the main effects of hand position and exposure condition, as well as for the interaction between these variables. $d f$, degrees of freedom; CE, mean constant error across participants; VE, mean variable error (i.e., the mean of standard errors) across participants; $x$, results in the $x$ direction (perpendicular to the mirror); $y$, results in the $y$ direction (parallel with the mirror).

hand hypothesis (that the visual information concerning arm position available via the reflection of a rubber hand is sufficient to increase the visual bias of reaching under conditions of visual-proprioceptive conflict) and contrast with the predictions of both the visual object hypothesis and the real hand/bilateral proprioceptive hypothesis.

There was a bias in reaching movements in the wooden block conditions corresponding to an underreach of about $17 \%$ of the lateral distance to the target. This residual bias is slightly larger than the bias observed in previous control conditions involving no wooden block or no mirror reflection at all (range, 8\%-15\%; Holmes et al., 2004; Holmes $\&$ Spence, 2005). There were no significant effects of the visual exposure condition on variable reaching error, indicating that participants were equally precise across experimental conditions, with their reaching movements biased in a constant manner according to the direction and size of the visual-proprioceptive conflict.

Our results suggest that the mechanism responsible for updating the felt position of the hand prior to reaching movements only has access to very basic information concerning the hand, which probably includes its shape and approximate orientation, since the reflection of a rubber hand was as effective as that of a real hand in altering reaching movements. However, even basic visual aspects of the rubber hand such as the color of its skin and its gender (and therefore its size and visual similarity relative to the average participant's hand) were not important factors in influencing reaching movements (separate betweenparticipants ANOVAs that included gender and skin color, which are not reported here, showed no significant effects of these variables on the interaction between the visual exposure condition and left hand position variables). Before accepting these conclusions, however, several other possible explanations for the altered reaching movements need to be ruled out.

First, it is possible that the visual bias of reaching associated with a rubber hand in Experiment 1 was due to the rubber hand being a more visually interesting object than the block of wood. That is, participants may simply have paid more attention to, or concentrated more upon, the available visual information (at the expense of proprioceptive information) in the real-hand and rubber hand conditions than they did in the wooden block condition. Second, the position of a participant's real right hand in the first experiment was not constant across conditions: In the real-hand condition, it was placed $15 \mathrm{~cm}$ to the right of the mirror, but in both the rubber hand and wooden block conditions, it was placed about $30 \mathrm{~cm}$ to the right of the mirror. It is possible that this difference in the posture of the right hand may have contributed in some manner to the visual bias of reaching in Experiment 1.

\section{EXPERIMENT 2}

In order to rule out the alternative possibilities discussed above, we conducted a second experiment. On the basis of previous experimental findings (see, e.g., Ehrsson et al., 2004; Graziano, Cooke, \& Taylor, 2000; Pavani et al., 2000), we hypothesized that simply changing the orientation of the rubber hand, making it incompatible with that of the real hand, would affect the hypothesized visually induced reaching bias: A rubber hand placed in the same orientation as the participant's own reaching hand (i.e., palm facing down) would increase the constant endpoint error both in comparison with a rubber hand in an orientation incompatible with the reaching hand (i.e., palm facing up) and with a wooden block. We assumed that a rubber hand in a palm up posture should be visually as interesting or attention-capturing as a palm down rubber hand, therefore controlling for a possible confounding effect of this visual interest factor. Furthermore, in Experiment 2, only one position for the participant's real nonreaching hand was used across all experimental conditions, to control for any potentially confounding effects of the posture of the right hand on reaching movements made with the left hand. In Experiment 2, participants were never exposed to the reflection of their own right hand during the experiment. Finally, it 
is also possible that the mirror illusion or the rubber hand illusion is itself an attention-capturing phenomenon and may have led to an increased reliance on visual information in planning and executing the reaching movement. We therefore decided to characterize any subjective aspects of the mirror conflict illusion and to attempt to correlate those aspects with any reaching biases, by administering a rubber hand illusion questionnaire similar to, and adapted from, those used elsewhere (see, e.g., Botvinick \& Cohen, 1998; Ehrsson et al., 2004; Pavani et al., 2000).

\section{Method}

Experiment 2 was identical to Experiment 1 except for the following details.

Participants. Twenty-one new participants (19-40 years of age, 16 female, 1 left-handed by self-report) were recruited.

Design. The visual exposure conditions included both the rubber hand and wooden block conditions. A new condition was also added to replace the real-hand condition of Experiment 1; in this new condition, the rubber hand was placed palm up on the table. In addition, a 10-item questionnaire was constructed. It consisted of a modified version of Botvinick and Cohen's (1998) questionnaire, which concerned any changes experienced during the experiment in the felt position, movement, identity, or ownership of the objects or of the participant's own hands. Questions were arranged vertically on the questionnaire sheet in a randomized order between participants but in the same order between conditions within participants.
Procedure. In Experiment 2, the participants never viewed their own hand, which was placed behind the paper screen throughout the experiment. After the experiment, the questionnaire was given to each participant to complete. Responses were recorded on a 7-point scale ranging from strongly disagree to strongly agree. These responses were assigned numerical values from -3 (strongly disagree) to +3 (strongly agree), treated as parametric data, and correlated with the regression slope data, as used in the original rubber hand illusion experiments (Botvinick \& Cohen, 1998). Differences in the questionnaire data between experimental conditions were assessed with repeated measures ANOVAs. Details of the questions asked in this questionnaire are provided in Figure 4. The participants were informed before the experiment that they would be asked to complete a questionnaire, but they were not informed that the questionnaire concerned subjective ratings of the rubber hand or mirror illusions.

\section{Results}

Constant error. The reaching error data are presented in the upper panel of Figure 3, and the ANOVA statistics in Table 1. The overall mean reaching error was $-2.5 \pm$ $0.2 \mathrm{~cm}$, and there was no main effect of visual exposure condition on this general rightward error (rubber hand palm down, $-2.3 \pm 0.3 \mathrm{~cm}$; rubber hand palm up, $-2.5 \pm$ $0.3 \mathrm{~cm}$; wooden block, $-2.8 \pm 0.3 \mathrm{~cm}$ ). This general bias was $0.9 \mathrm{~cm}$ larger than, but not significantly different from, the general rightward error in Experiment 1 [betweenexperiments ANOVA, $F(1,37)=2.42, p=.13]$. As in Ex-
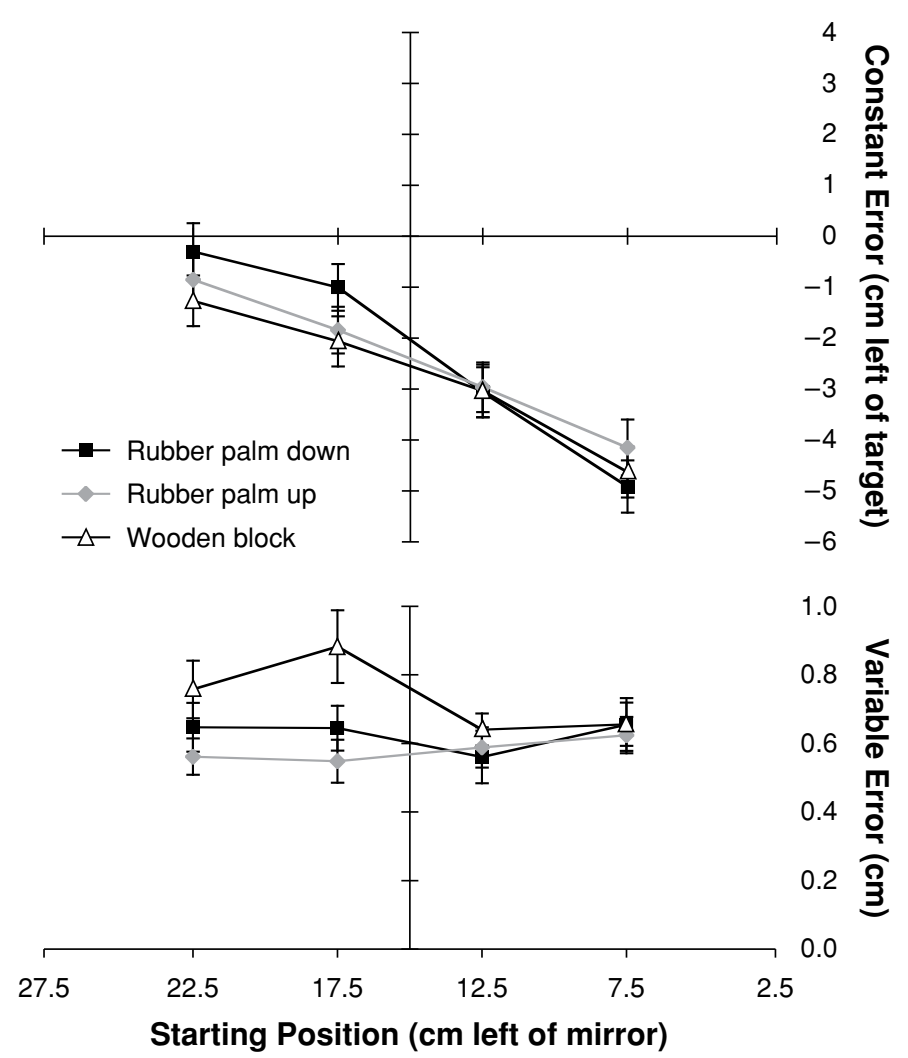

Figure 3. Mean ( $\pm S E$ ) endpoint errors for reaching movements in Experiment 2. Top: Constant reaching error $(M \pm S E$ of the individual mean reaching errors). Bottom: Variable reaching error ( $M \pm S E$ of the standard error of the individual mean reaching errors). 
periment 1 , reaching movements were biased more by the rubber hand in a palm down posture $(31.8 \% \pm 3.1 \%)$ than by the block of wood $(22.1 \% \pm 3.0 \%)$. The novel finding to emerge from Experiment 2 was that exposure to the incompatible rubber arm $(22.0 \% \pm 3.7 \%)$ also resulted in less bias of reaching movements than did the compatible rubber arm. Separate ANOVAs comparing pairs of conditions confirmed that reaching behavior in the rubber hand condition was significantly different from that in both the rubber palm up condition [exposure condition, $F(1,20)=$ $0.08, p=.79$; left hand position, $F(3,60)=59.3, p<$ .001 ; left hand position $\times$ exposure condition interaction, $F(3,60)=7.49, p<.001]$ and the wooden block condition [exposure condition, $F(1,20)=0.66, p=.42$; left hand position, $F(3,60)=78.4, p<.001$; left hand position $\times$ exposure condition interaction, $F(3,60)=5.30$, $p<.005]$. There was no significant difference between reaching in the latter two conditions [exposure condition, $F(1,20)=0.28, p=.61$; left hand position, $F(3,60)=$ $49.5, p<.001$; left hand position $\times$ exposure condition interaction, $F(3,60)=0.45, p=.72]$.

Variable error. There was a significant main effect of visual exposure condition, but no other significant terms in the ANOVA of the variable error data arising from Experiment 2. Variable error was higher in the wooden block condition $(0.74 \pm 0.05 \mathrm{~cm})$ than in either the rubber hand palm down $(0.63 \pm 0.05 \mathrm{~cm})$ or the rubber hand palm up $(0.58 \pm$ $0.04 \mathrm{~cm}$ ) condition. Visual inspection of Figure 3 reveals that this result was primarily due to increased variability in the two leftmost starting positions for this condition.

Rubber hand illusion questionnaire ratings. Of the 10 questionnaire items (see Figure 4), only two showed ratings that were significantly positively correlated with the magnitude of the reaching bias across all three conditions.
They were (1) "The rubber hand [wood] in the mirror was my left hand" ( $\left.r^{2}=.16, p<.01\right)$; and (2) "The rubber hand [wood] on the table was my right hand" $\left(r^{2}=.14\right.$, $p<.01)$. The mean rating for the rubber hand condition was never positive (i.e., on average, participants always disagreed with the statements), suggesting that there were no strong or consistent components of the rubber hand illusion in the present experiments, as one might expect, since no attempts were made either to induce or encourage participants to experience the rubber hand illusion.

Ratings from all 10 questions across all three conditions were entered into a multiple linear regression analysis, with the percentage reaching error (i.e., the magnitude of the bias) as the dependent variable and the 10 questionnaire ratings as predictor variables for each participant. Overall, the proportion of variance in the percentage reaching error explained by the subjective ratings was low $\left(r^{2}=.27\right)$, and taken together, the subjective ratings did not significantly predict the reaching errors, although they did approach significance $[F(10,52)=1.90, p=$ .07]. Analyzing each question separately with a one-way ANOVA revealed only 5 questions in which a significant effect of visual exposure condition on the subjective ratings was found. The results of these additional statistical tests are displayed in Figure 4. Post hoc comparisons between individual conditions for each question revealed that the majority of the significant differences between conditions arose from comparisons between the rubber hand and wooden block conditions (5 out of 10 comparisons, $p<.05$ ). By contrast, the critical comparisons between the rubber hand palm down and palm up conditions revealed only one significant difference in the subjective ratings ("I was surprised the rubber hand [in the mirror] didn't move when I moved my left hand," $p<.05$ ). Finally,

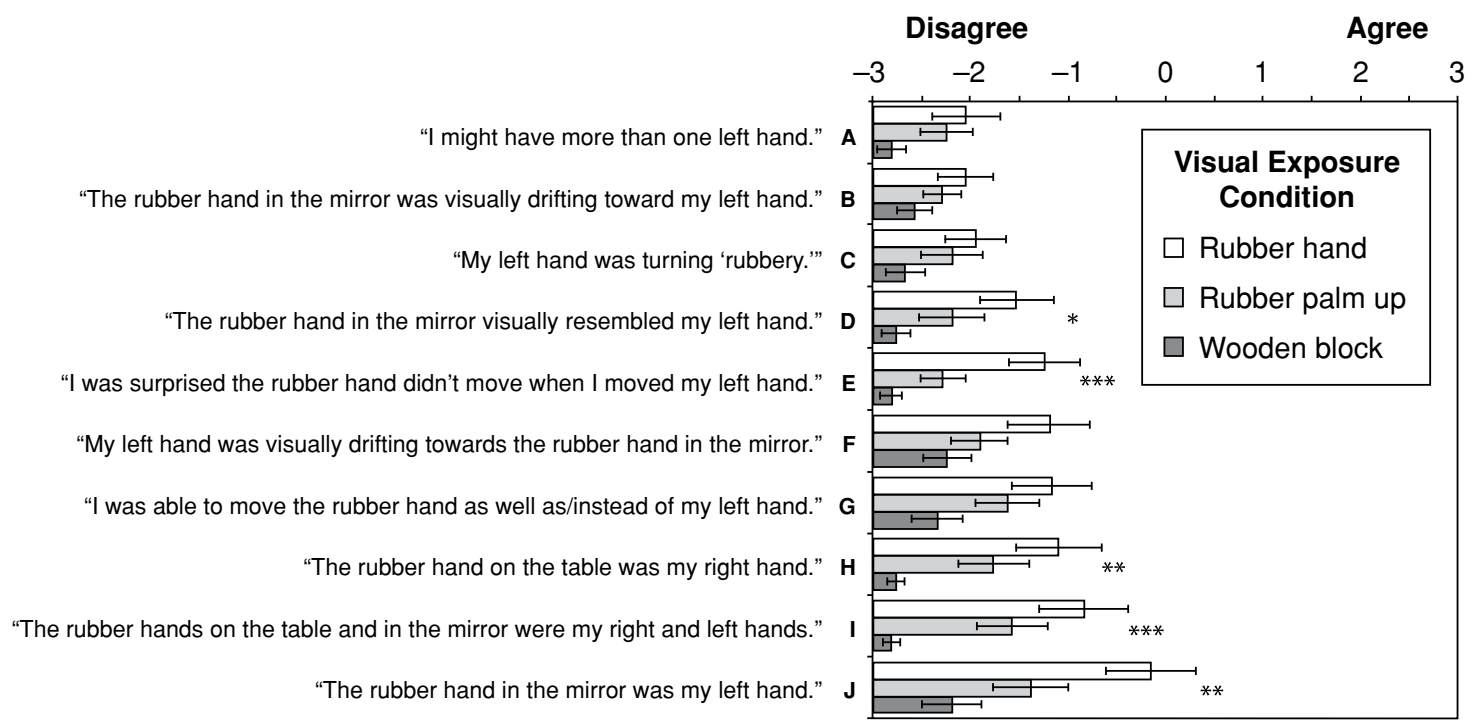

Figure 4. Questionnaire results showing the ratings of subjective aspects of the rubber hand illusion during the visual exposure period. The questions are arranged vertically in ascending order of the mean rating of agreement reported in the rubber hand condition. Asterisks indicate significant differences in one-way ANOVAs between conditions for each question considered separately: ${ }^{*} p<.05 ;{ }^{* *} p<.01$; ${ }^{* * *} p<.001$. The bars and error bars show mean $\pm S E$. 
only one of the comparisons between the rubber palm up and wooden block conditions reached significance ("The rubber hands on the table and in the mirror were my right and left hands," $p<.05$ ).

\section{Discussion}

The analysis of the reaching data from Experiment 2 shows that changing the posture of the visible rubber hand from incompatible to compatible (with respect to the real reaching hand) significantly affected participants' reaching behavior. This result suggests that one major determinant of the rubber-hand-induced visual bias of proprioception is the posture of the visible hand itself (see also Graziano et al., 2000; Pavani et al., 2000). Furthermore, it suggests that the processes responsible for the recalibration of proprioception by vision have access, and are sensitive, to visual information concerning the posture (palm up vs. palm down) of the hand seen in the mirror. From previous research on human participants and macaque monkeys, we know that gross changes in the posture of a visible arm (such as turning the arm by $90^{\circ}$ or $180^{\circ}$ so the hand points medially, laterally, or facing toward the body) have significant effects on multisensory behavior and multisensory neural interactions. Here, we show that, similar to the changes in neural firing in macaque area 5 (Graziano et al., 2000), more subtle posture changes, such as rotating the arm around its longitudinal axis, can also induce changes in simple reaching behaviors.

The analysis of the questionnaire data revealed only rather weak correlations between the subjective illusion measures and the reaching error data. The only questionnaire items that were significantly correlated with the reaching errors were those that related to the feeling that the rubber hands in the mirror and on the table were the participants' real left and right hands, respectively. First, this result suggests that following visual exposure to the rubber hand, the subjective rubber hand illusion was not particularly evident, at least on average for the participants tested here, according to the answers they gave (several participants did indeed feel strong illusory components of the rubber hand illusion, but as is quite common in rubber hand illusion studies, the strength of the illusory effects varied considerably across participants; see, e.g., Botvinick \& Cohen, 1998; Ehrsson et al., 2004). Second, this result suggests that at least part of the effect on subsequent reaching errors of the rubber hand seen in the mirror could be due to the participants' feeling that the rubber hand was their right hand on the table, rather than their left hand behind the mirror. The rubber hand was seen both indirectly in the mirror, and directly, in participants' peripheral vision. It is not clear, therefore, which of a participant's hands was more important in generating the postexposure effects on reaching errors.

\section{EXPERIMENT 3}

In Experiments 1 and 2, the posture of a participant's own right and left hands was always prone (palm down). Following the results of Experiment 2, in which the posture of the rubber hand significantly influenced participants' reaching behavior, it is important to rule out any possible influence of the posture of each participant's unseen right hand on the reaching movements made with the unseen left hand. Furthermore, it seemed important to test whether changes in the visible posture of the participant's own right, nonreaching hand also have a significant effect on reaching movements made with the left hand. In our third experiment, therefore, we manipulated the posture of the participant's right hand (palm down vs. palm up) and the identity of the hand seen in the mirror (prone rubber hand vs. real hand) in a factorial manner. If the posture of the participant's right hand has a significant effect on reaching movements, regardless of whether that hand was seen in the mirror or hidden away behind the screen, then there must be some role for bilateral postural information in the visual bias of reaching demonstrated in Experiments 1 and 2 (the bilateral postural hypothesis). Alternatively, if the posture of the participant's right hand only has an effect on reaching behavior when that hand is visible in the mirror (and not when a rubber hand is seen palm down in the mirror during exposure to both real-hand postures), then we can conclude that the visual bias of reaching is due to visual factors alone and can discount the influence of bilateral postural information (the visual hypothesis).

\section{Method}

Experiment 3 was identical to Experiment 1, except for the following details.

Participants. Twenty-four participants (18-32 years of age, 17 female, 1 left-handed by self-report) were recruited. Six participants had already participated in Experiment 1, and 4 had participated in Experiment 2. The remaining participants had not taken part in any mirror box experiments previously.

Design. There were four visual exposure conditions, composed of the combination of the factors right hand posture (palm down vs. palm up) and visible hand (right hand vs. palm down rubber hand viewed in the mirror). The four visual exposure conditions were therefore: (1) real palm down (real right hand visible in the mirror, with a palm down posture); (2) real palm up (real right hand visible in the mirror, with a palm up posture); (3) rubber hand, real palm down (rubber hand visible in the mirror with a palm down posture, real right hand hidden behind the paper screen with a palm down posture); (4) rubber hand, real palm up (rubber hand visible in the mirror with a palm down posture, real right hand hidden behind the paper screen with a palm up posture). The visual exposure conditions were presented in four blocks of 20 trials each, in a fully counterbalanced order across participants (i.e., 24 different block orders). The exposure duration was reduced to $10 \mathrm{sec}$ per trial, to keep the overall length of the experiment to around $30 \mathrm{~min}$ while maintaining the number of trials per condition.

\section{Results}

Constant error. The reaching error data are displayed in Figure 5. They were entered into a three-way ANOVA with the variables left hand position $(7.5,12.5,17.5$, and $22.5 \mathrm{~cm}$ ), right hand posture (palm down vs. palm up), and visible hand (real right hand vs. rubber hand). The ANOVA revealed a significant main effect of left hand position $[F(3,69)=96.94 ; p<.001]$ but no significant main effect of right hand posture $[F(1,23)=0.06, p=.82]$ or visible hand $[F(1,23)=2.16, p=.16]$. Of the two-way 

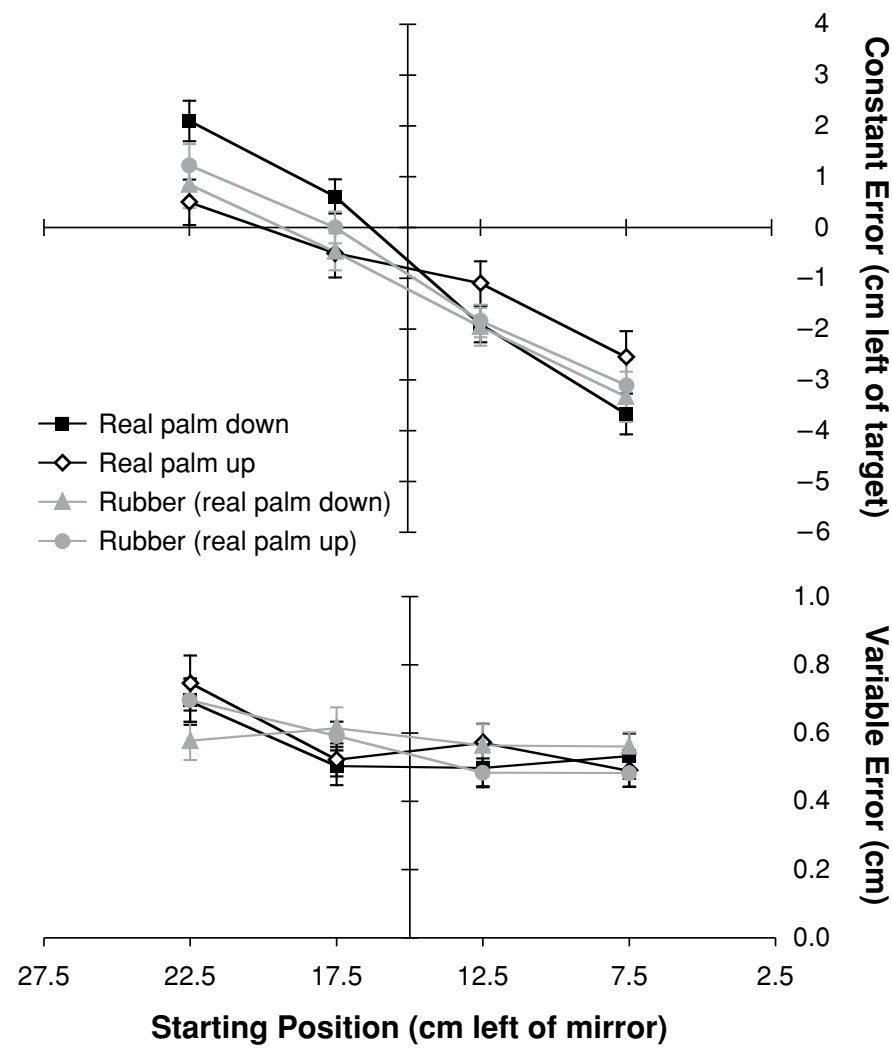

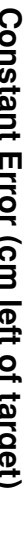

Figure 5. Mean ( $\pm S E$ ) endpoint errors for reaching movements in Experiment 3. Top: Constant reaching error $(M \pm S E$ of the individual mean reaching errors). Bottom: Variable reaching error $(M \pm S E$ of the standard error of the individual mean reaching errors).

interactions, only the interaction between left hand position and right hand posture was significant $[F(3,69)=$ $17.73, p<.001]$, revealing a stronger dependence overall on the initial position of the left hand when the real right hand was in a palm down posture. The critical test concerned the three-way interaction between left hand position, right hand posture, and visible hand. This interaction was significant $[F(3,69)=19.20, p<.001]$, revealing that the interaction between the left hand position and the right hand posture depended upon the visible hand (i.e., whether the posture of the right hand was visible or not). Two separate two-way ANOVAs were conducted on the reaching error data for the rubber hand present and rubber hand absent conditions. This analysis revealed that when the participant's real right hand was visible in the mirror, the interaction between left hand position and right hand posture was significant $[F(3,69)=35.74, p<.001]$, but when the rubber hand in a palm down posture was visible in the mirror, there was no significant interaction between left hand position and right hand posture $[F(3,69)=0.54$, n.s.]. In short, the posture of the participant's right hand only influenced reaching behavior when it was visible in the mirror, and not when it was hidden behind the screen.

The mean endpoint error overall was $-0.9 \pm 0.1 \mathrm{~cm}$ to the right of the target position. The mean overall errors for each condition were as follows: Real hand palm down, $-0.7 \pm 0.3 \mathrm{~cm}$; real hand palm up, $-0.9 \pm 0.3 \mathrm{~cm}$; rubber hand (real palm down), $-1.2 \pm 0.3 \mathrm{~cm}$; rubber hand (real palm up), $-0.9 \pm 0.3 \mathrm{~cm}$. The dependence of the mean terminal reaching error on the initial left hand position was stronger in Experiment 3 than in Experiments 1 and 2. Expressed as a percentage, reaching errors were $39.7 \% \pm 2.7 \%$ in the real palm down condition, $19.5 \% \pm$ $3.2 \%$ in the real palm up condition, $28.0 \% \pm 4.0 \%$ in the rubber hand (real palm down) condition, and $29.7 \% \pm$ $3.1 \%$ in the rubber hand (real palm up) condition.

Variable error. The ANOVA on the variable error data revealed a significant effect of left hand position $[F(3,69)=8.0, p<.001]$ but no other significant effects or interaction terms. As in Experiments 1 and 2, variable error was highest for reaches made from the leftmost starting position (for $7.5 \mathrm{~cm}, 0.51 \pm 0.03 \mathrm{~cm}$; for $12.5 \mathrm{~cm}, 0.53 \pm 0.03 \mathrm{~cm}$; for $17.5 \mathrm{~cm}, 0.56 \pm 0.03 \mathrm{~cm}$; for $22.5 \mathrm{~cm}, 0.68 \pm 0.04 \mathrm{~cm}$ ).

\section{Discussion}

The results of Experiment 3 confirmed that there was no detectable influence of the posture (palm down vs. palm up) of the participant's unseen and nonreaching right hand unless that hand was visible in the mirror. This 
result supports the visual hypothesis and contradicts the bilateral postural hypothesis, suggesting that the enhanced reaching biases we have observed following vision of the rubber hand are an exclusively visual phenomenon, with no significant contribution from postural information relating to the unseen right hand.

The size of the reaching bias following exposure to the rubber hand was smaller than that following exposure to the real hand in Experiment 3. In Experiment 1, there was no significant difference between these two conditions. There are several possible reasons for this, the most likely being between-participants variability in the effect of the rubber hand on reaching biases. Although almost every participant we have tested in a series of mirror reaching experiments has shown an effect of vision of their real hand, the effects of the rubber hand are strong but somewhat less consistent between individuals; some have shown no effect at all, and others have shown stronger effects for exposure to the rubber hand than to their real hand. It is also possible that the number of experimental conditions (four) in Experiment 3 may have decreased the effects of the rubber hand relative to the real hand, if one supposes that the illusory effects of the rubber hand may be overcome through practice. This possibility was tested explicitly with an additional ANOVA on the data from the two rubber hand conditions alone (collapsed), with the between-participants factor block order (1-4). This revealed no significant effect or interactions involving the block order term $[F(3,44)=1.11, p=.36]$, further suggesting that no order effects were present in our data.

During the peer review process, it was suggested that the differences between the rubber hand conditions and the wooden block conditions might be due to some strategic difference or differences in the orienting or allocation of attention either during the visual exposure period or during reaching toward the target itself. Although we have not yet tested this possibility explicitly, the results of Experiments 1-3 lead us to believe that this possibility is unlikely. First, any strategic or attentional effects would have to produce results exactly in line with the effects of visual exposure duration, relative position, and hand posture that we have demonstrated in our present and previous experiments (see also Holmes et al., 2004; Holmes \& Spence, 2005). Second, such changes in the allocation of attention (for example, away from the target or from performance of the reaching task) ought to result in significant increases in variable reaching error between experimental conditions. As the analysis of variable error in Experiments 1-3 has shown, this was not the case: Reaching was equally precise in all visual exposure conditions, except for Experiment 2 , in which the wooden block condition showed less precise reaching (higher variable error) than did the rubber hand conditions - an effect opposite in direction from that predicted by either the decreased attention or the distraction argument.

Nevertheless, the possibility remains that during the 10- to 12-sec exposure period, the presence of the rubber hand in a posture compatible with that of the participant's real hand (and perhaps the experience of the mirror or rubber hand illusion itself) was in some way attentionally capturing, which led to differences in constant reaching error across conditions. Indeed, the rubber hand illusion ratings were significantly higher (though on average they still negated the presence of the illusion) in the compatible rubber hand conditions than in the incompatible hand and wooden block control conditions, suggesting that the subjective aspects of these two conditions are quite different. To attempt to control for these more subjective aspects of the experimental manipulations, we performed two further experiments in which participants were required to perform an additional task during the exposure period. In Experiment 4, we asked participants to perform two similar finger-tapping tasks during the visual exposure period, and in Experiment 5 they were asked to perform a visual discrimination task during the exposure period.

\section{EXPERIMENT 4}

We reasoned that if the between-conditions differences in reaching behavior in Experiments 1-3 were due not to the effects of visual exposure to a hand but rather to attentional or concentration differences, providing an additional active task in which participants needed to pay attention to both left (unseen) and right (seen) hands simultaneously might control for such strategic differences. We also reasoned that if we could devise two similar tasks that differed in their visual-proprioceptive congruence with respect to the apparent unseen hand (i.e., the mirror reflection) and the real unseen hand (the left hand hidden behind the mirror), any differences in reaching behavior must therefore be due to the visual-proprioceptive congruence rather than to the performance of the secondary task per se.

The reasoning above requires us to assume that the two tasks are sufficiently similar to each other not to induce different task-dependent shifts of attention. We chose to use a finger-tapping task for this purpose (see also Holmes et al., 2004; Holmes \& Spence, 2005). We asked participants to tap the index fingers of both hands at approximately $1 \mathrm{~Hz}$ while viewing the moving index finger of the right hand in the mirror reflection. Participants were asked to tap their left and right fingers either in phase with each other (i.e., both the left and the right fingers tapping the table at the same time), or $180^{\circ}$ out of phase with each other (i.e., the left finger reaching the upper limit of the tapping movement while the right finger contacts the table and vice versa). To preserve the visual-proprioceptive congruence during the in-phase tapping task and to maximize the incongruence in the out-of-phase task, we did not use the rubber hand or wooden block as an exposure object in Experiment 4 (since it was not possible to control the finger movements of the rubber hand!). Instead, participants viewed their own hands in the mirror in three experimental conditions: In-phase movements (synchronous tapping), out-of-phase movements (asynchronous tapping), and passive exposure (no tapping). This design allowed us to determine whether the performance of a secondary task per se was qualitatively independent from the basic visual bias of reaching effect that we are arguing for. 
If the performance of both finger tapping tasks influenced the reaching bias equally and resulted in a significantly different reaching bias in comparison with the passive visual exposure condition alone, then we can conclude that performing any secondary task affects reaching behavior (the distraction hypothesis). If, by contrast, the synchronous finger tapping task induced a significantly greater reaching bias than did the asynchronous task, then we can conclude that it is the congruence between the visually specified hand seen in the mirror and the actual state of the hand behind the mirror that is crucial for the enhanced reaching bias effects (the visuomotor-proprioceptive congruence hypothesis). From our previous results (Holmes \& Spence, 2005), and in accordance with the latter hypothesis, we predicted that the synchronous tapping task should induce a greater reaching bias than would the passive exposure task.

\section{Method \\ Participants. Twelve new participants (19-40 years of age, 7 female, all right-handed by self-report) were recruited. \\ Design. There were three visual exposure conditions: synchro- nous tapping; asynchronous tapping; and passive visual exposure. \\ Procedure. Experiment 4 required several minor modifications to the design and apparatus of Experiments 1-3. First, no rubber hand or wooden block was used. In all three visual exposure condi- tions, the participants placed their right hand $15 \mathrm{~cm}$ from the mir- ror, on the right hand position mark. They were asked to tap the index fingers of their two hands at approximately $1 \mathrm{~Hz}$ (i.e., one tap per second per index finger). In the synchronous tapping condition, the apparent rhythm of tapping was therefore $1 \mathrm{~Hz}$ (both fingers tapping at the same time, approximately once per second), and in the asynchronous condition it appeared to be $2 \mathrm{~Hz}$ (a different finger tapping approximately every half second). This apparent doubling in frequency was highlighted to the participants, in order to attempt to keep the number of taps per finger constant across exposure condi- tions. The participants did not report having any problems in pro- ducing these tapping movements. The experimenter monitored the participants and gave occasional prompts to adjust their tapping fre- quency, but the primary concern was that the participants maintain in-phase or out-of-phase tapping in the appropriate experimental conditions. The finger tapping itself was not recorded and served only as a secondary active task during the visual exposure period. The passive visual exposure condition was identical to the real-hand (palm down) conditions of Experiments 1 and 3.}

\section{Results}

Constant error. The mean constant error across left hand position and visual exposure condition is presented in the upper panel of Figure 6, and the ANOVA statistics are presented in Table 1 . The mean reaching error overall was $1.0 \pm 0.3 \mathrm{~cm}$ to the right of the target. The three experimental conditions produced comparable overall mean reaching errors (synchronous tapping, $-1.2 \pm 0.5 \mathrm{~cm}$; passive [no tapping], $-1.1 \pm 0.4 \mathrm{~cm}$; asynchronous tapping, $-0.8 \pm 0.4 \mathrm{~cm})$. The dependence of the constant reaching error on the left hand starting position differed significantly between the visual exposure conditions. Reaches in the synchronous-tapping condition were most biased (by $38.1 \% \pm 3.2 \%$ ), followed by those in the passive exposure condition $(35.5 \% \pm 3.2 \%)$, and performance in the asynchronous tapping condition was least affected $(24.8 \% \pm 2.1 \%)$. Separate ANOVAs confirmed that reaching errors differed significantly between the synchronous and asynchronous conditions [exposure condition $\times$ left hand position interaction, $F(3,33)=10.95$, $p<.001]$ and between the passive and asynchronous conditions $[F(3,33)=8.37, p<.001]$, but they were not significantly different between the synchronous and passive conditions $[F(3,33)=0.74, p=.54]$.

Variable error. The lower panel of Figure 6 and Table 1 show the results of the analysis of the variable error data. There were no significant main effects or interactions, though the main effect of left hand position approached significance; this trend was due to the higher variability for reaching movements made from the leftmost starting position, consistent with the results of Experiments 1-3.

\section{Discussion}

The results of Experiment 4 extend and qualify those of Experiments 1-3 by showing that, following exposure to the visual image of one's own hand, reaching movements are strongly influenced only if the visual information concerning the apparent left hand is congruent with proprioceptive and motor information from the real left hand hidden behind the mirror. If the visible hand in the mirror is tapping out of phase with the real hand behind the mirror, subsequent reaching behavior is less biased by the conflicting visual information concerning hand position. Experiment 4 therefore suggests an important role for visuomotor-proprioceptive correlation in the generation of the bias of reaching following visual exposure to mirror-reflected hands. However, although such active synchronous bimanual tapping is sufficient to produce a strong bias of reaching, it is not necessary; reaching was biased equally during the passive visual exposure and the synchronous tapping conditions.

These results suggest that the performance of a secondary task by itself does not influence the reaching bias following visual exposure to the mirror-reflected hand, unless that task involves introducing an incongruence between what is seen in the mirror and what the hand behind the mirror is doing. These results therefore run counter to the distraction hypothesis and support the visuomotorproprioceptive congruence hypothesis instead.

Before we accept this conclusion, however, it is also possible that in the asynchronous tapping condition participants paid more attention to the proprioceptive information available from the unseen left hand, which resulted in the decreased reaching bias shown in that condition compared with the other two conditions, in which participants were in general biased more toward the visual information by such strategic or task-dependent differences. In addition, and particularly in relation to the rubber hand and wooden block conditions of Experiments 1-3, it is also possible that there is something specific to the two visual exposure objects (the rubber hand and the wooden block), or perhaps to the residual illusory experience of viewing rubber hands, that necessarily entails that participants devote more visual attention to the rubber hand and less to the wooden block during the exposure period. Such enhanced visual attention in the rubber hand versus the 


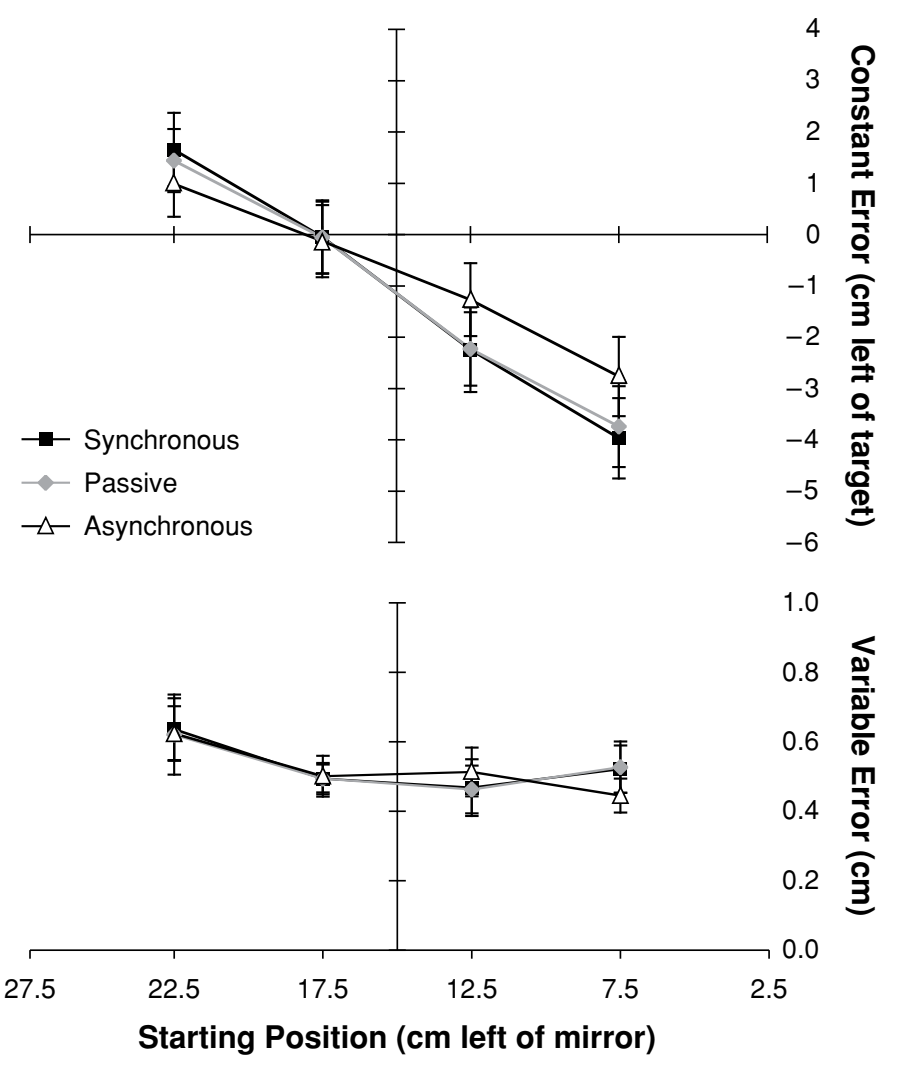

Figure 6. Mean $( \pm S E)$ endpoint errors for reaching movements in Experiment 4. Top: Constant reaching error $(M \pm S E$ of the individual mean reaching errors). Bottom: Variable reaching error $(M \pm S E$ of the standard error of the individual mean reaching errors).

wooden block conditions might therefore lead to greater dependence on the (incorrect) visual information concerning hand position, and less dependence on the (correct) proprioceptive information. Providing a secondary task during the exposure period that requires sustained visual attention and vigilance and is identical between visual exposure conditions could test such a possibility. To address these questions concerning the allocation of visual attention, we therefore performed one final experiment.

\section{EXPERIMENT 5}

If the rubber hand condition leads to an increased dependence on, or attention toward, visual information than does the wooden block condition, then participants in the former condition should be faster and make fewer errors responding selectively to a visual target during the prereach exposure period than they do in the latter (the enhanced visual attention hypothesis). Alternatively, under this hypothesis, if performance on the secondary task is identical between the visual exposure conditions, there should also be no significant difference in reaching behavior. By contrast, if the presence of the rubber hand does not enhance attentional allocation to the visual information in contrast with the wooden block, then there should be no differences in performance on the secondary task, but the directional reaching bias should still be stronger in the rubber hand than in the wooden block condition (the automatic visual bias hypothesis).

\section{Method}

Experiment 5 was similar to Experiments 1-3, except for the following details.

Participants. Twelve new participants were recruited (1819 years of age, 10 female, 1 left-handed by self-report).

Apparatus and Materials. A red LED $5 \mathrm{~mm}$ in diameter was positioned immediately behind the index finger of the rubber hand or the wooden block so that it was visible to participants only in the mirror and not directly. The LED served as a visual fixation point, and its illumination provided a visual target stimulus. A foot pedal was placed beneath the participant's left foot, and another foot pedal was operated by the experimenter. The LED and the foot pedals were connected via a parallel port interface box to a PC operating custom software programmed in the Turbo Pascal programming language.

Design. There were two visual exposure conditions: the rubber hand (palm down) condition of Experiments 1-3 and the wooden block condition of Experiments 1 and 2. The participants never viewed their own right hand in the mirror. The exposure duration was shortened from 12 to $10 \mathrm{sec}$ to allow a greater number of trials to be performed within a single 30 -min experimental session. There were 8 trials per visual exposure condition and left hand position $(2$ exposure conditions $\times 4$ left hand positions $\times 8$ trials $=64$ trials 
per participant). The visual exposure conditions were run in separate blocks, with the order counterbalanced across participants. The four left hand positions were presented in a pseudorandomized order, determined by the computer prior to each block of trials.

Procedure. Each trial began when the experimenter depressed and released the pedal under his foot. The participants were instructed to fixate on the LED during the 10 -sec prereach exposure period. At a random point in time between 3 and $7 \mathrm{sec}$ after the beginning of each trial, the LED flashed either once (for $200 \mathrm{msec}$ ), or twice ( $65 \mathrm{msec}$ on, $70 \mathrm{msec}$ off, and $65 \mathrm{msec}$ on). The participants were instructed to respond only to the double flashes by lifting their left foot as quickly as possible off the foot pedal (i.e., a visual discrimination/go-no-go task). After $8.5 \mathrm{sec}$ from the beginning of the trial, a tone ( $400 \mathrm{~Hz}, 500 \mathrm{msec}$ in duration) was presented from the computer. The participants were instructed to make a single eye movement from the visual fixation LED to the target location (i.e., as seen in the mirror) and to prepare to reach. After a random delay of between 1 and $2 \mathrm{sec}$, a second tone $(1 \mathrm{kHz}, 2,000 \mathrm{msec}$ in duration) sounded and acted as the reach cue. The participants then made a single, smooth reaching movement to place their left index finger as accurately as possible on the target. The experimenter marked the reaching endpoint location (in the middle and immediately in front of the index fingertip) on millimeter-squared graph paper using a different-colored pen for each left hand position. Each participant was given eight practice trials before the start of the experiment; verbal feedback about reaching accuracy was provided during these practice trials, and the experimenter moved the participant's finger to the target location for additional tactile/proprioceptive feedback. No further feedback was provided.

\section{Results}

Constant error. In Experiment 5, unlike Experiments $1-4$, we also performed an analysis on errors in the frontback $(y)$ direction. The mean terminal errors in the leftright $(x)$ direction are displayed in Figure 7, and the relevant ANOVA statistics are presented in Table 1. Overall mean reaching error in the rubber hand condition $(0.3 \pm$ $0.4 \mathrm{~cm}$ rightward and $1.0 \pm 0.3 \mathrm{~cm}$ behind the target from the participants' perspective) was slightly, but not significantly, lower than in the wooden block condition $(0.9 \pm$ $0.4 \mathrm{~cm}$ rightward and $0.7 \pm 0.4 \mathrm{~cm}$ behind the target). As predicted, the dependence of the reaching errors in the $x$ direction on the left hand position was stronger in the rubber hand condition (mean error $=30.1 \% \pm 5.0 \%$ ) than in the wooden block condition $(16.0 \% \pm 4.1 \%)$. There were no significant effects or interactions involving the visual exposure condition for errors in the $y$-direction.

Variable error. In the left-right $(x)$ direction, there was a significant effect of left hand position on the variable reaching error, with the leftmost starting position showing higher variable error than the other positions (for $7.5 \mathrm{~cm}$, $0.53 \pm 0.04 \mathrm{~cm}$; for $12.5 \mathrm{~cm}, 0.47 \pm 0.03 \mathrm{~cm}$; for $17.5 \mathrm{~cm}$, $0.56 \pm 0.39 \mathrm{~cm}$; for $22.5 \mathrm{~cm}, 0.63 \pm 0.05 \mathrm{~cm})$. There were no other significant terms. For the front-back $(y)$ direction, there were no significant terms in the ANOVA.
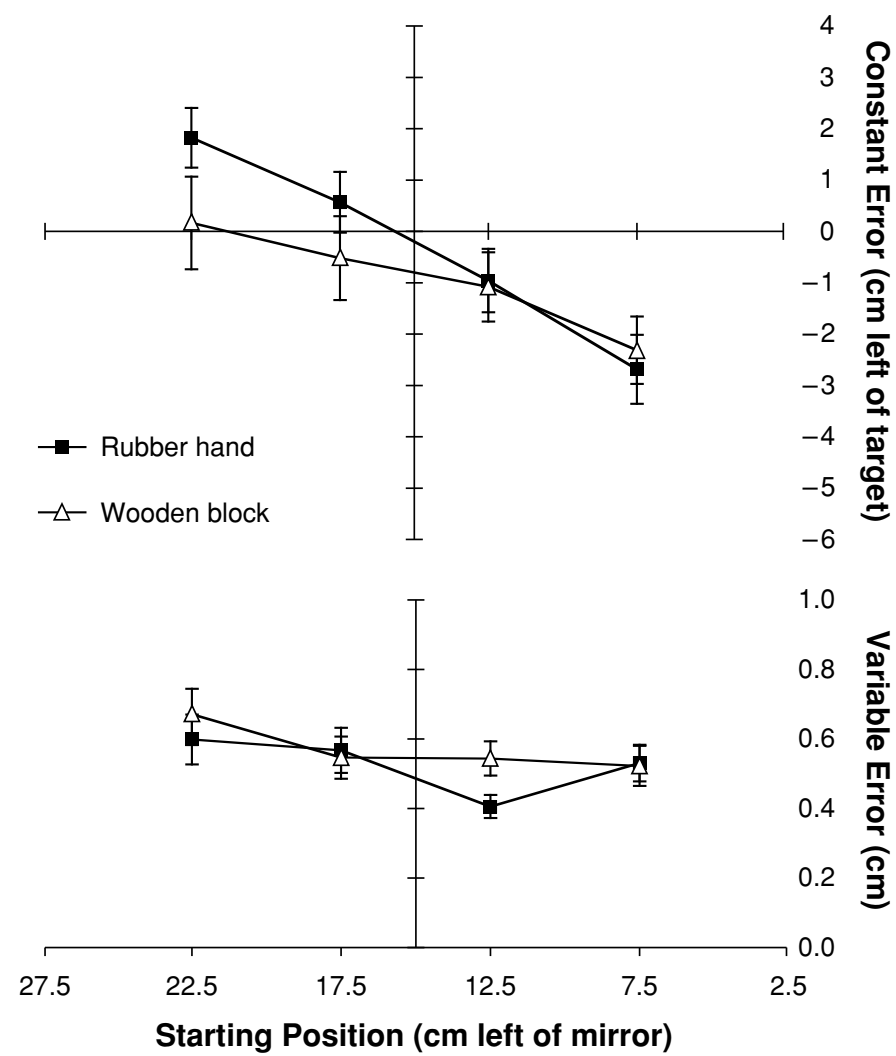

Figure 7. Mean $( \pm S E)$ endpoint errors for reaching movements in Experiment 5. Top: Constant reaching error $(M \pm S E$ of the individual mean reaching errors). Bottom: Variable reaching error ( $M \pm S E$ of the standard error of the individual mean reaching errors). 
Visual discrimination task. Performance on the visual discrimination (go-no-go) task was assessed using a within-participants repeated measures ANOVA; the reaction time and error data, broken down by left hand position and visual exposure condition, are presented in Figure 8 . The mean overall reaction time was $685 \pm 15 \mathrm{msec}$, and there were no significant differences between overall performance on the two exposure conditions in reaction times (rubber hand, $684 \pm 22 \mathrm{msec}$; wooden block, $685 \pm$ $22 \mathrm{msec}$ ), number of targets missed (rubber hand, 3.9\% \pm $0.9 \%$; wooden block, $3.1 \% \pm 0.9 \%$ ), or false alarms (rubber hand, $0.0 \% \pm 0.0 \%$; wooden block, $0.8 \% \pm 0.6 \%$ ). The ANOVA revealed no significant terms.

\section{GENERAL DISCUSSION}

Experiment 1 showed that $12 \mathrm{sec}$ of visual exposure to the reflection of the participant's own right hand induced significant biases in subsequent reaching movements of the left hand, and that this bias was equal in magnitude when vision of a rubber right hand was substituted for vision of the real hand. Visual exposure to both real and rubber hands produced more bias than did visual exposure to a block of wood. Experiment 2 replicated, clarified, and extended this finding by showing that the posture of the rubber hand with respect to the participant's real hand behind the mirror significantly influenced the reaching bias. When the rubber hand was misaligned with the real hand (i.e., when it was placed palm up), there was no difference in reaching behavior between the effects of exposure to the rubber hand and the effects of exposure to the block of wood. However, a rubber hand aligned compatibly with the participant's hand induced significantly more bias. The analysis of the questionnaire data revealed only two subjective aspects of the experience of the rubber-hand-in-mirror situation that accounted for a significant proportion of the variance in the reaching data above the $5 \%$ significance level. These factors related to the subjective feelings that the rubber hands in the mirror and on the table were one's real left and right hand, respectively. Experiment 3 showed that manipulating the posture of the participant's nonreaching hand only affected the behavior of the reaching hand when the nonreaching hand was visible in the mirror (thus supporting the visual hypothesis) and had no effect when an artificial hand was visible in a congruent posture in the mirror (arguing against the bilateral proprioceptive hypothesis). We also showed that performance of an additional task during the prereach exposure period only affected reaching bias when the task altered the visuomotor-proprioceptive correlation between the apparent (mirror) and real (hidden) hands (Experiment 4); it did not affect bias when the secondary task concerned a visual discrimination/go-no-go task (Experiment 5).

Throughout the five experiments reported here, there were no effects of block order on the size or direction of the reaching movements, and there were no obvious effects of trial-by-trial practice in Experiment 5 (data refereed but not reported here). These results suggest that whatever process(es) lead to the reaching biases, they are not dependent on practice with or experience of the task, but are highly replicable across experiments, participants, blocks, and trials. Furthermore, and perhaps surprisingly, there were no significant effects of gender or skin color on the interaction between hand position and visual exposure condition (again, data refereed but not reported here). The latter result may be surprising given that the rubber hand we used was similar to that of a small Caucasian female's

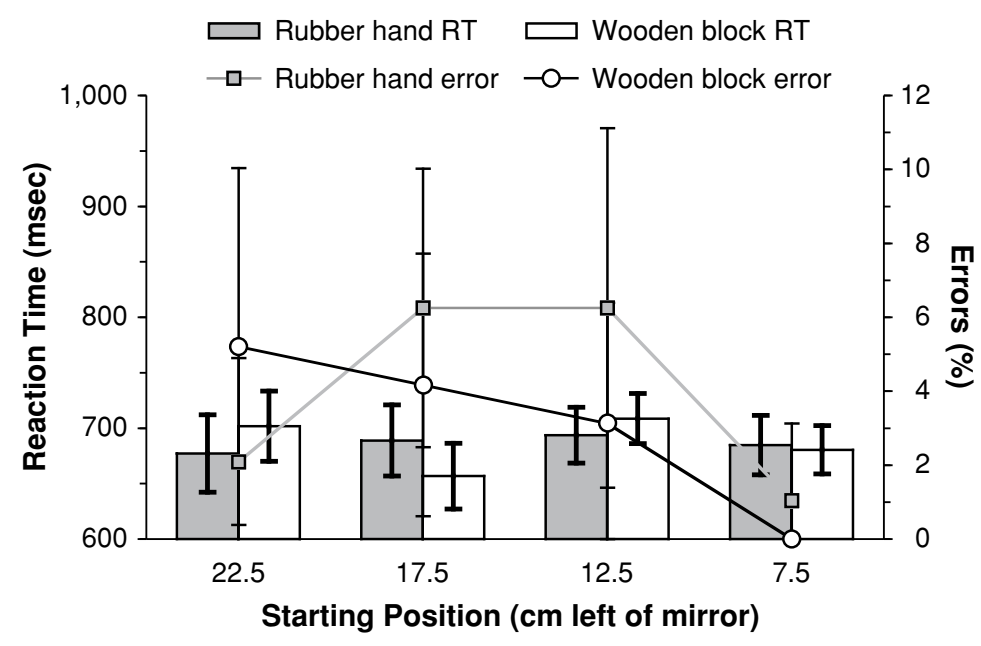

Figure 8. Performance on the visual discrimination/go-no-go task in Experiment 5. The gray columns and filled gray squares indicate mean ( $\pm S E$ ) RTs and percent errors (both misses and false alarms), respectively, in the rubber hand condition. The white columns and open circles indicate mean ( $\pm S E$ ) RTs and percent errors (both misses and false alarms), respectively, in the wooden block condition. 
hand, yet the participants varied widely in height and skin color, though the majority were female (61 of 87 , or $70 \%$ ). The lack of such participant-specific effects, however, underlines our assertion that whatever process is responsible for the reported reaching bias, it has access primarily only to approximate visual information concerning the location of the hand and is less sensitive to fine visual details of any particular visible hand.

The finding that the posture of the rubber hand was the crucial factor for increasing the bias of reaching with respect to the wooden block control condition is compatible with several other recent findings. First, Graziano et al. (2000) found that about a third of cells in macaque monkey area 5 showing tonic activity related to the positions of the right and/or left arms were also sensitive to the position of a stuffed monkey arm placed in view and near the animal's real arm (which was placed out of sight). More importantly, certain cells were also sensitive to the orientation of the hand and the identity of the hand; the position and posture of a fake right hand modulated the firing of area 5 cells that preferred right arm postures more than it did the firing of those cells with a preference for left arm postures.

Arm posture dependent changes in multisensory processing following exposure to misaligned or artificial hands have also been demonstrated in brain-damaged patients (Farnè et al., 2000; Rorden et al., 1999) and normal human participants (Austen et al., 2004; Pavani et al., 2000). Furthermore, the influence of the posture of the rubber hand and the presence of synchronous versus asynchronous multisensory stimulation of the real hand have both been shown to modulate activity in brain areas closely related to the multisensory representation of the body (Ehrsson et al., 2004; see also Lloyd, Shore, Spence, \& Calvert, 2003). Ehrsson et al. found that activity in the premotor cortex was most closely correlated with the experience of the rubber hand illusion under the combination of synchronous visual-tactile stimulation and a compatible posture of the rubber hand with respect to the real hand. Activity in the posterior parietal cortex, by contrast, was enhanced both by synchronous multisensory stimulation and by the compatible orientation of the artificial hand, but was less correlated with the rubber hand illusion itself.

In conjunction with previous results from our laboratory (Holmes et al., 2004; Holmes \& Spence, 2005), the present results imply that passive visual exposure to artificial hands in a congruent posture induces a visual recalibration of proprioception of the participant's real hand position toward the position of the artificial hand (in the present experiments, toward the virtual position of the rubber hand). It is also possible, as suggested by one of our reviewers, that the effects of the hand-position- and posture-dependent manipulations we have reported here might be due to an alteration of visuospatial processing rather than of the felt azimuthal location of the reaching limb. Although we cannot definitively rule out this possibility with the present data alone, a recalibration of visual space seems to us to be a far less likely explanation for our results than a recalibration of proprioceptive information, particularly since the participants' gaze direction, the visual location of the exposure object, and the target location were constant and not manipulated across experimental conditions and experiments. Proprioceptive signals relating to hand position are known to decay in the absence of visual information within a very short time scale of only about $15 \mathrm{sec}$, an interval similar to the exposure durations used here (Wann \& Ibrahim, 1992).

Effects such as those demonstrated in the present research probably depend at least partly on multisensory processes that integrate vision and proprioception in the posterior parietal cortex, which forms part of the reaching motor circuit (i.e., the medial intraparietal cortex or parietal reach region, and the premotor cortex; see Connolly, Andersen, \& Goodale, 2003; Ehrsson et al., 2004; see also Lloyd et al., 2003). We are currently using transcranial magnetic stimulation to test the hypothesis that the superior parietal lobule (areas 5 and 7 of the posterior parietal cortex) plays a role in the visual guidance of reaching movements in the mirror illusion experiment. Of particular interest to us will be to examine kinematic aspects of the reaching movements using a three-dimensional positiontracking system, in order to determine whether reaching movements starting from a position in which vision and proprioception are in conflict are different from those in the absence of conflict, as well as whether such differences evolve over the course of the reaching movement. Several of the participants in the present experiments commented, for instance, that the initiation of reaching movements felt more difficult after exposure to the rubber hand, and that the rubber hand "paralyzed" them in some strange manner. Such strange sensations of ownership and agency have been reported before in similar experiments (see, e.g., Jackson \& Zangwill, 1952; Nielsen, 1963; Sullivan, 1969).

Our results further suggest that the process of visual recalibration of proprioception by artificial hands can, to a certain extent, be dissociated from the experience of the rubber hand illusion and the "ownership" of sensations applied to the rubber hand. More importantly, reaching biases following exposure to rubber hands occur somewhat automatically, in the absence of any conscious attempt to induce (on the part of the experimenter) or to experience (on the part of the participant) any subjective aspects of the rubber hand illusion. Passive visual exposure alone induces a significant reaching bias without any strong accompanying illusory sensations of ownership of the rubber hand (see also Pavani et al., 2000, in which participants also, on average, disconfirmed the presence of the rubber hand illusion). This finding suggests that reaching or proprioceptive biases are not reliable objective measures of the rubber hand illusion itself, and that proprioceptive recalibration in fact may be either a necessary prerequisite for the rubber hand illusion to occur or a process causally unrelated to illusory experiences of bodily ownership.

\section{Conclusions}

Our brains compute the position of our hands based on a weighted sum of visual and proprioceptive information (Haggard, Newman, Blundell, \& Andrew, 2000; van 
Beers, Wolpert, \& Haggard, 2002). In the absence of visual information, proprioception is relied upon to guide the trajectory of subsequent reaching movements. When visual information concerning the apparent position of the hand is provided by a mirror reflection of the other hand, the brain cannot help but integrate that information with the available proprioceptive information. We have shown here that even the somewhat rudimentary visual information provided by passive vision of an artificial hand, placed in a plausible anatomical alignment with the participant's real hand, can bias subsequent reaching movements as much as vision of the real hand itself can, yet without inducing any strong illusory sensations of ownership of the artificial hand. We suggest that those areas of the brain responsible for integrating visual and proprioceptive information only have access to very basic visual information concerning body parts such as the hand. This visual information may specify only the approximate shape, size, and position of the hand, yet it may still be sufficient to begin the process of recalibrating the felt position of the hand toward the visually specified apparent position.

\section{REFERENCES}

Altschuler, E. L., Wisdom, S. B., Stone, L., Foster, C., Galasko, D., LleWEllyn, D. M., \& Ramachandran, V. S. (1999). Rehabilitation of hemiparesis after stroke with a mirror. Lancet, 353, 2035-2036.

Armel, K. C., \& Ramachandran, V. S. (2003). Projecting sensations to external objects: Evidence from skin conductance response. Proceedings of the Royal Society of London: Series B, 270, 1499-1506.

Austen, E. L., Soto-Faraco, S., Enns, J. T., \& Kingstone, A. (2004). Mislocalizations of touch to a fake hand. Cognitive, Affective, \& Behavioral Neuroscience, 4, 170-181.

BÉDARd, P., \& Proteau, L. (2001). On the role of static and dynamic visual afferent information in goal-directed aiming movements. Experimental Brain Research, 138, 419-431.

Botvinick, M., \& Cohen, J. (1998). Rubber hands "feel” touch that eyes see. Nature, 391, 756.

BurnetT, C. T. (1904). Studies in the influence of abnormal position upon the motor impulse. Psychological Review, 11, 370-394.

Connolly, J. D., Andersen, R. A., \& Goodale, M. A. (2003). fMRI evidence for a "parietal reach region" in the human brain. Experimental Brain Research, 153, 140-145.

Desmurget, M., Rossetti, Y. K. K., Jordan, M., Meckler, C., \& Prablanc, C. (1997). Viewing the hand prior to movement improves accuracy of pointing performed toward the unseen contralateral hand. Experimental Brain Research, 115, 180-186.

Ehrsson, H. H., Spence, C., \& Passingham, R. E. (2004). That's my hand! Activity in premotor cortex reflects feeling of ownership of a limb. Science, 305, 782-783.

Farnè, A., Pavani, F., Meneghello, F., \& LÀdavas, E. (2000). Left tactile extinction following visual stimulation of a rubber hand. Brain, 123, 2350-2360.

Franz, E. A., \& Packman, T. (2004). Fooling the brain into thinking it sees both hands moving enhances bimanual spatial coupling. Experimental Brain Research, 157, 174-180.

Ghilardi, M. F., Gordon, J., \& GHEz, C. (1995). Learning a visuomotor transformation in a local area of workspace produces directional biases in other areas. Journal of Neurophysiology, 73, 2535-2539.

Graziano, M. S. A., Cooke, D. F., \& Taylor, C. S. R. (2000). Coding the location of the arm by sight. Science, 290, 1782-1786.

Haggard, P., Newman, C., Blundell, J., \& Andrew, H. (2000). The perceived position of the hand in space. Perception \& Psychophysics, 62, 363-377.

Holmes, N. P., Crozier, G., \& Spence, C. (2004). When mirrors lie: "Visual capture" of arm position impairs reaching performance. Cognitive, Affective, \& Behavioral Neuroscience, 4, 193-200.
Holmes, N. P., \& Spence, C. (2005). Visual bias of unseen hand position with a mirror: Spatial and temporal factors. Experimental Brain Research, 166, 489-497.

JACKSON, C. V., \& ZANGWILl, O. L. (1952). Experimental finger dyspraxia. Quarterly Journal of Experimental Psychology, 4, 1-10.

Kennett, S., Taylor-Clarke, M., \& Haggard, P. (2001). Noninformative vision improves the spatial resolution of touch in humans. Current Biology, 11, 1188-1191.

Lloyd, D. M., Shore, D. I., Spence, C., \& Calvert, G. A. (2003). Multisensory representation of limb position in human premotor cortex. Nature Neuroscience, 6, 17-18.

Newport, R., Hindle, J. V., \& JACKson, S. R. (2001). Links between vision and somatosensation: Vision can improve the felt position of the unseen hand. Current Biology, 11, 975-980.

Niebauer, C. L., Aselage, J., \& Schutte, C. (2002). Hemispheric interaction and consciousness: Degree of handedness predicts the intensity of a sensory illusion. Laterality, 7, 85-96.

Nielsen, T. I. (1963). Volition: A new experimental approach. Scandinavian Journal of Psychology, 4, 225-230.

Pavani, F., Spence, C., \& Driver, J. (2000). Visual capture of touch: Out-of-the-body experiences with rubber gloves. Psychological Science, 11, 353-359.

Peled, A., Pressman, A., Geva, A. B., \& Modai, I. (2003). Somatosensory evoked potentials during a rubber-hand illusion in schizophrenia. Schizophrenia Research, 64, 157-163.

Peled, A., Ritsner, M., Hirschmann, S., Geva, A. B., \& Modai, I. (2000). Touch feel illusion in schizophrenic patients. Biological Psychiatry, 48, 1105-1108.

Ramachandran, V. S., \& Rogers-Ramachandran, D. (1996). Synaesthesia in phantom limbs induced with mirrors. Proceedings of the Royal Society of London: Series B, 263, 377-386.

RAMACHANDRAN, V. S., Rogers-RAMACHANDRAN, D., \& CobB, S. (1995). Touching the phantom limb. Nature, 377, 489-490.

Ro, T., Wallace, R., Hagedorn, J. C., Farnè, A., \& Pienkos, E. (2004). Visual enhancing of tactile perception in posterior parietal cortex. Journal of Cognitive Neuroscience, 16, 24-30.

Rorden, C. R., Heutink, J., Greenfield, E., \& Robertson, I. H. (1999). When a rubber hand "feels" what the real hand cannot. NeuroReport, 10, 135-138.

Rossetti, Y. K. K., Desmurget, M., \& Prablanc, C. (1995). Vectorial coding of movement: Vision, proprioception, or both? Journal of Neurophysiology, 74, 457-463.

Rossetti, Y. K. K., Stelmach, G. E., Desmurget, M., Prablanc, C., \& JEANNEROD, M. (1994). The effect of viewing the static hand prior to movement onset on pointing kinematics and variability. Experimental Brain Research, 101, 323-330.

Sarlegna, F., Blouin, J., Bresciani, J., Bourdin, C., Vercher, J., \& Gauthier, G. M. (2004). Target and hand position information in the online control of goal-directed arm movements. Experimental Brain Research, 151, 524-535.

Sathian, K., Greenspan, A. I., \& Wolf, S. L. (2000). Doing it with mirrors: A case study of a novel approach to neurorehabilitation. Neurorehabilitation \& Neural Repair, 14, 73-76.

Sullivan, R. (1969). Experimentally induced somatagnosia. Archives of General Psychiatry, 20, 71-77.

TAstevin, J. (1937). En partant de l'expérience d'Aristotle: Les déplacements artificiels des parties du corps ne sont pas suivis par le sentiment de ces parties ni par les sensations qu'on peut y produire [Starting from Aristotle's experiment: Artificial displacements of parts of the body are not followed by feelings in these parts or by the sensations which can be produced in them]. L'Encephale, 1, 57-84, 140-158.

TSAKIRIS, M., \& HAGGARD, P. (2005). The rubber hand illusion revisited: Visuotactile integration and self-attribution. Journal of Experimental Psychology: Human Perception \& Performance, 31, 80-91.

van Beers, R. J., Wolpert, D. M., \& Haggard, P. (2002). When feeling is more important than seeing in sensorimotor adaptation. Current Biology, 12, 834-837.

Wann, J. P., \& Ibrahim, S. F. (1992). Does limb proprioception drift? Experimental Brain Research, 91, 162-166.

(Manuscript received December 30, 2004; revision accepted for publication July 26, 2005.) 